\title{
Risk Assessment of Plan Schedule by Monte Carlo Simulation
}

\author{
Zhaoni Kong ${ }^{1, a}$, Jianping Zhang ${ }^{1, b^{*}}$, Chao $\mathrm{Li}^{1}$, Xiaoying Zheng ${ }^{1}$ and Qingqing \\ Guan $^{2}$
}

${ }^{1}$ Faculty of Civil Engineering and Architecture, Kunming University of Science and Technology, P.R. China

${ }^{2}$ Faculty of Environmental Science and Engineering, Kunming University of Science and Technology, P.R. China

a237071828@qq.com, b1181557223@qq.com

Keywords: risk assessment, Monte Carlo simulation, triangular distribution, schedule control.

Abstract: With an increasingly complex and rapidly changing construction circumstance, owners and their contractors are confronting challenges from risk management while maintaining control and improving performance. With great capabilities for project management solution, Monte Carlo simulation was used to forecast projects' execution results. In this paper, a practical example, construction and installation engineering in cargo area of Kunming Changshui international airport project is studied to show explicitly the benefits of using Monte Carlo simulation to assess risk in practical scheduling.

\section{Introduction}

Plan schedule is one of the most important responsibilities of construction project management. A success in managing a project requires a complete and realistic project schedule that plans and coordinates various activities. Also, a reasonable schedule should predict the completion and milestone dates of the project. But reasonable project schedule is difficult to achieve because the rules of project scheduling is unclear to the scheduler with unrealistic deadlines, uncertainty of activity durations [1]. No two activities among projects are exactly same, thus even well experienced project managers could not precisely estimate the completion of a project. Moreover modern construction projects are often complex in characteristics. There are unforeseen reasons that make the majority of construction projects often overrun scheduled completion time and even miss targets [2]. Laufer \& Howell [3] find that about $80 \%$ projects have high degree of uncertainty at the beginning of constructions. More and more project managers therefore begin to realize the importance of uncertainties in project schedule. Challenged by unforeseen reasons, project managers begin to treat risk management as their routine requirement.

Project Management Institute defined a project risk as 'an uncertain event or condition that, if it occurs, has a positive or negative effect on at least one project objective' [4]. As can been seen, a risk has two parts--probability of occurrence and the effect of each occurrence. Risk management can be categorized as technical, external, internal and organizational work. The process of risk management includes risk identification, risk assessment, risk response and risk documentation [5]. Modeling and quantifying risk are crucial parts of risk management.

In 1950s, quantitative network modeling techniques were used to simulate the construction process. Network diagram techniques can find activity relationships, and help the establishment of durations and coordination of construction projects. However, Birrell [6] noticed that the critical path method (CPM) uses a single value to estimate each activity and even the entire construction without considering the effects of uncertainty. CPM assumes that each activity has only one possible outcome and will be completed successfully. In fact, there are numerous possible results for an activity with different consequences. Besides, in most cases the probability of activity durations calculated by critical path methodology (CPM) to achieve the plan was less than 25\%. Recently, Hulett [1] found program evaluation and review technique (PERT) also always underestimated schedule risk, ignoring the possible risk that may be caused by PERT-slack paths. 
PERT only focused on the one longest or PERT-critical path. According to Bonny [7] and Li et al. [8], activity durations calculated by PERT were almost always less than actual statistic mean with deviation up to $30 \%$. Besides, traditional scheduling processes ignore the uncertain and risky nature of the environment of a project. So, network-based planning processes have been proven inadequate to conduct practical risk analysis in scheduling. To produce an effective schedule, a more thorough scheduling process is required with formal recognition and assessment of project uncertainty.

To improve accuracy, brainstorming, sensitivity analysis, the Delphi method, or decision tree analysis are also used as typical existing analyzing risk methods. Recently, as a useful modeling technique to monitor risk, Monte Carlo simulation has great potential and the convenience [9]. Many results show that Monte Carlo simulation produces the correct answers without abovementioned flaws. As a modern way to evaluate project schedule risk, Monte Carlo simulation can overcome the deterministic limitations of traditional scheduling processes. In this paper, a practical example is studied to show explicitly the benefits of using Monte Carlo simulation to assess risk in practical scheduling.

\section{Simulation and results}

\section{Monte Carlo simulation}

Monte Carlo simulation was developed in the 1940s and became available under the help of personal computers and associated software, e.g. Primavera Risk, Primavera Crystal Ball, @ RISK for Projects and so on. Monte Carlo has been involving in many industrial, scientific, logistical and social fields in many years. Recently project managers have used Monte Carlo to simulate the project completion date and the project cost estimate. Through computing the CPM schedule many times, Monte Carlo simulation can determine the project completion date on the basis all possible combinations of the uncertain activity durations.

In Monte Carlo simulation, the project model is computed many times (iterated) with the input values (e.g., activity durations) chosen at random for each iteration from the probability distributions of these variables. Monte Carlo simulation follows certain steps [10]
a. Creation of the quantitative model of the form of $\mathrm{y}=f\left(\mathrm{x}_{1}, \mathrm{x}_{2}, \ldots, \mathrm{x}_{\mathrm{n}}\right)$,
b. Selection of the random variables $\mathrm{x}_{\mathrm{i} 1}, \mathrm{x}_{\mathrm{i} 2}, \ldots, \mathrm{x}_{\mathrm{in}}$,
c. Evaluation of the model and output storage in $y_{i}$,
d. Repeat steps (b) and (c) as many times needed (for $\mathrm{i}=1$ to $\mathrm{k}$ ),
e. Analysis of results.

\section{A case study}

A practical case, construction and installation engineering in cargo area of Kunming Changshui international airport project is presented in this section to illustrate the proposed method. In this scenario, the Oracle Crystal Ball software was used to analyze the schedule, while it was assumed that schedule values follow a triangular distribution with the $99 \%$ confidence interval. In order to meet the abovementioned requirement of accuracy, the number of simulation should be greater or equal to 4096 [11]. So, the number of runs was set to 10,000 .

To improve probability of activity durations, the Monte Carlo simulation actually allows the recalculation of the model over and over again, each time using different randomly selected sets of values from the input probability distributions, while using all valid combinations of the possible inputs, in order to simulate all possible outcomes. The spreadsheet of stochastic scheduling model and relevant Gantt chart can be found in Table 1 and Figure 1. 


\begin{tabular}{|c|c|c|c|c|c|c|c|c|c|c|c|c|}
\hline Task & $\begin{array}{l}\text { Predecesso } \\
\mathbf{r}\end{array}$ & $\begin{array}{l}\text { Duration } \\
\text { Optimisti } \\
\text { c }\end{array}$ & $\begin{array}{l}\text { Duration } \\
\text { Expected }\end{array}$ & $\begin{array}{l}\text { Duration } \\
\text { Pessimist } \\
\text { ic }\end{array}$ & $\begin{array}{l}\text { Sim } \\
\text { Value }\end{array}$ & EST & EFT & LST & LFT & Slack & $\begin{array}{l}\text { Crit } \\
\text { Pat } \\
\text { h }\end{array}$ & $\begin{array}{l}\text { Prob. } \\
\text { On } \\
\text { CP }\end{array}$ \\
\hline \multicolumn{13}{|l|}{ Start } \\
\hline $\mathbf{A}$ & Start & 103 & 153 & 199 & 153 & 0 & 153 & 0 & 153 & 0 & 1 & $\begin{array}{l}74.51 \\
\%\end{array}$ \\
\hline B & A & 44 & 59 & 65 & 59 & 153 & 212 & 153 & 212 & 0 & 1 & $\begin{array}{l}74.51 \\
\%\end{array}$ \\
\hline C & B & 49 & 61 & 70 & 61 & 212 & 273 & 212 & 273 & 0 & 1 & $\begin{array}{l}74.51 \\
\%\end{array}$ \\
\hline D & Start & 152 & 182 & 233 & 182 & 0 & 182 & 30 & 212 & 30 & 0 & $\begin{array}{l}25.49 \\
\%\end{array}$ \\
\hline $\mathbf{E}$ & D & 53 & 61 & 69 & 61 & 182 & 243 & 212 & 273 & 30 & 0 & $\begin{array}{l}25.49 \\
\%\end{array}$ \\
\hline $\mathbf{F}$ & $\mathrm{C}, \mathrm{E}$ & 73 & 92 & 109 & 92 & 273 & 365 & 273 & 365 & 0 & 1 & $100 \%$ \\
\hline G & $\mathrm{F}$ & 49 & 61 & 73 & 61 & 365 & 426 & 395 & 456 & 30 & 0 & $2.54 \%$ \\
\hline H & G I & 80 & 121 & 161 & 121 & 456 & 577 & 486 & 607 & 30 & 0 & $2.61 \%$ \\
\hline
\end{tabular}

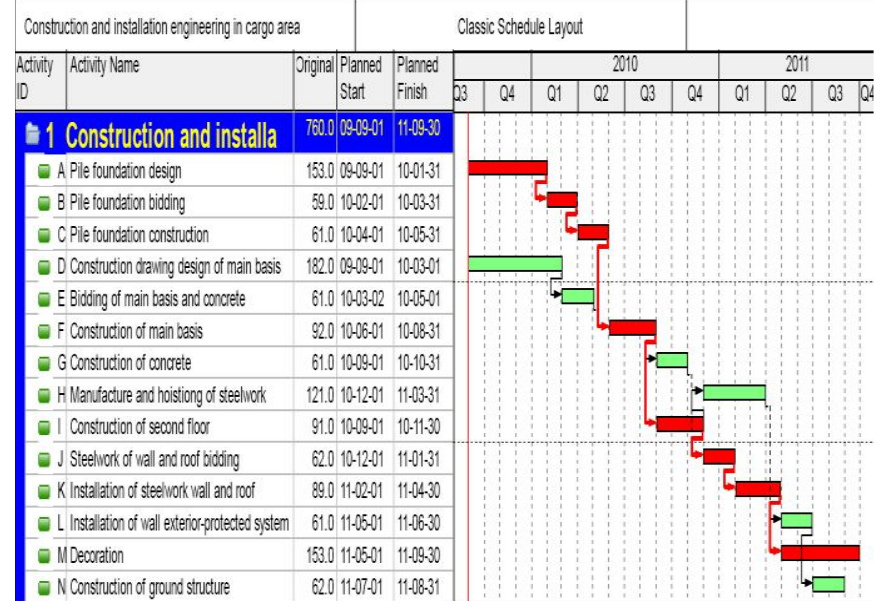

Figure 1. Gantt chart containing the determinis tic time [Oracle® Primavera ${ }^{\circledR}$ P6]

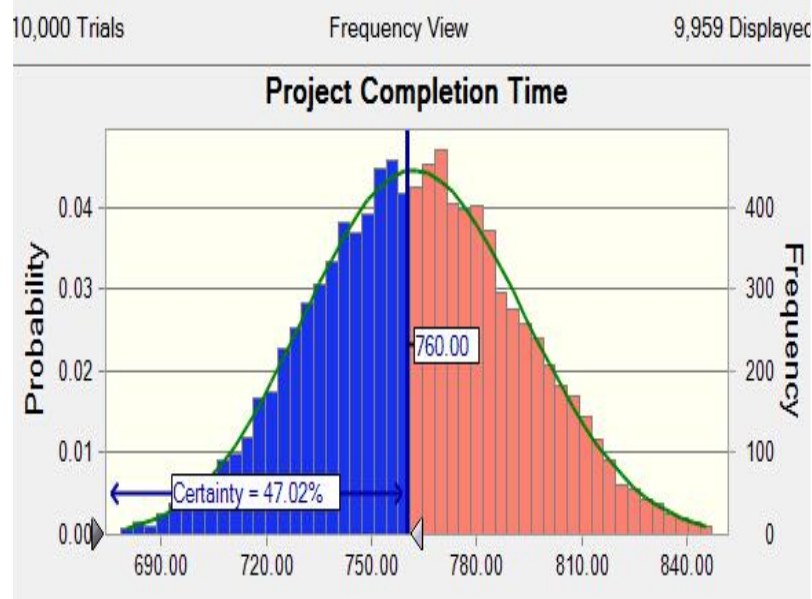

Fig. 2 Frequency view

As is shown in Fig. 2, axis $\mathrm{X}$ depicts the completion date and axis $\mathrm{Y}$ stands for the probability of the given value to occur. The blue-colored area represents the positive values of the project completion time and the pink-colored area represents the negative values. It plots the distribution curve of all of the dates when the project is going to end and replace a firm date with a range of dates. Fig. 2 also exhibits that the probability that the completion date of September 30, 2011 can be met is only 47.02\%. Thus, the completion date calculated in CPM scheduling is not very likely to occur, given the risks that have been specified.

Since there is only about $47.02 \%$ likelihood that this project can be finished on September 30, 2011 or earlier, the probability of schedule completion on this project without risk mitigation is problematic. It should be noted that the date of September 30 is not even the most likely completion date for this project. From the histograms, it seems that the most likely duration (the tallest column in Figure 2) is closer to 770 than to 760. This finding indicates that it is unwise to put confidence on the sum of even the most likely durations as a predictor of the duration. As a common value for a conservative target is a date that has an 80 percent probability of success, abbreviated often as ' $\mathrm{P}-80$ ', the table shows the results that $\mathrm{P}-80$ date is about 788.29 according to Table 2. 
Tab. 2 Percentiles

\begin{tabular}{ll}
\hline Percentile & Forecast values \\
\hline $\mathbf{0 \%}$ & 663.37 \\
$\mathbf{1 0 \%}$ & 724.23 \\
$\mathbf{2 0 \%}$ & 736.86 \\
$\mathbf{3 0 \%}$ & 746.59 \\
$\mathbf{4 0 \%}$ & 754.62 \\
$\mathbf{5 0 \%}$ & 762.64 \\
$\mathbf{6 0 \%}$ & 770.23 \\
$\mathbf{7 0 \%}$ & 778.52 \\
$\mathbf{8 0 \%}$ & 788.29 \\
$\mathbf{9 0 \%}$ & 802.15 \\
$\mathbf{1 0 0 \%}$ & 860.68 \\
\hline
\end{tabular}

Tab.3 Sensitivity Analysis

\begin{tabular}{ll}
\hline Activity Name & Percentage \\
\hline $\mathbf{A}$ & $25.2 \%$ \\
$\mathbf{M}$ & $24.7 \%$ \\
$\mathbf{I}$ & $14.6 \%$ \\
$\mathbf{K}$ & $13.4 \%$ \\
$\mathbf{J}$ & $11.3 \%$ \\
$\mathbf{F}$ & $5.9 \%$ \\
$\mathbf{D}$ & $2.1 \%$ \\
$\mathbf{B}$ & $1.7 \%$ \\
$\mathbf{C}$ & $0.8 \%$ \\
$\mathbf{E}$ & $0.2 \%$ \\
$\mathbf{L}$ & $0.1 \%$ \\
$\mathbf{N}$ & $0 \%$ \\
$\mathbf{H}$ & $0 \%$ \\
$\mathbf{G}$ & $0 \%$ \\
\hline
\end{tabular}

The risk analysis by Monte Carlo simulation also can identify the major risk and find a way to the unfavorable outcomes. In order to find the key factors that can impact the completion date, sensitivity analysis was conducted. Table 3 shows which factors have the most potential impact on the distribution of possible outcomes and how the variations in project's objectives correlate with variations in different uncertainties. From sensitivity analysis, it is clear that A (Design) has the highest uncertainty and impact on the distribution of possible outcomes. M (construction of second floor) and I (Decoration) also have high uncertainties and impacts, some due to long construction duration and key construction period.

In this scenario, it is necessary for a project manager to find causes of variances and take some corrective actions. To create a schedule contingency allowance, the move-in for the next available window would be postponed. If the project completion date is immovable, schedule should be compressed and improved with the expectation of 80 or 90 percent probability of completion. Although this compression always cause a general rise in price, risk assessment is not cost but an investment in terms of actual money saved, since resources are used more efficiently and the consequence of a delay to the project can be avoided.

Through Monte Carlo simulation, the project risks and their potential consequences can be identified for practicalcase. A balance between the precisely planning a project schedule and the scarce resources also can be achieved. The results confirm that risk assessment by Monte Carlo simulation can assist in planning decision, setting contingency time and taking corrective action.

\section{Conclusion}

As an advanced data mining method in project management, Monte Carlo simulation can provide wealthy informations, including the criticality path report, the criticality distribution, and the duration probability curves for risk management. As seen from a practical case, compared with firm date given by CPM, Monte Carlo simulation can provide a range of date for risk analysis. By Monte Carlo simulation, risk analysis can help refine the schedule, identify and monitor risk events, and respond with corrective actions in case of unfavorable outcomes. In short, Monte Carlo simulation can provide direct pictorial information to assist the decision makers in selecting a realistic yet acceptable project completion time.

\section{References}

[1] D. Hulett, Practical Schedule Risk Analysis, Farnham, Surrey, United Kingdom, 2009.

[2] K.G. Cooper, The $\$ 2,000$ hour: How managers influence project performance through the rework cycle, PM J. 4(1994) 11-24.

[3] A. Laufer, G.A. Howell, Construction planning: Revising the paradigm, PM J. 3(1993) 23-33. 
[4] Project Management Institute, A Guide to the Project Management Body of Knowledge, Project Management Institute, Pennsylvania, 2004.

[5] A. Basu, Practical risk analysis in scheduling, AACE International Transactions, West Virginia, 1998.

[6] G.S. Birrell, Construction planning-beyond the critical path, J. Constri. Div. ASCE. 106(1980) 389-407.

[7] J.B. Bonny, Handbook of construction management and organization, VanNostrand Reinhold Company, Vancouver, WA, 1973.

[8] R.G. Li, G.X.Wang, Y. Li, J.L. Ren, Study on uncertainty of activity duration in PERT, Systems Engineering and Electronics, 19(1997) 40-45(chinese).

[9] Y.Reuven, Simulation and the Monte Carlo method, Wiley, New York, 2008.

[10] D. Vose, Quantitative risk analysis, a guide to Monte Carlo simulation modelling, Wiley, New York, 1996.

[11] N. Duan, H.M. Xue, Y. Pan, A method for determining the number realizations in the calculation of reliability by Monte Carlo simulation method, Coal Mine Machinery, 3(2002) 13-14. 